\title{
TEORIA DA ESTRUTURA A TERMO DAS TAXAS DE JUROS
}

RESUMO

Este estudo usa um modelo intertemporal de equilíbrio geral de precificação de ativos para estudar a estrutura a termo das taxas de juros. Nesse modelo, expectativas, aversão ao risco, alternativas de investimento e preferências quanto ao momento do consumo atuam na determinação dos preços. Muitos fatores tradicionalmente mencionados como influentes sobre a estrutura a termo são, assim, incluídos de uma maneira plenamente condizente com o comportamento maximizador e as expectativas racionais. O modelo leva a fórmulas específicas de preços de debêntures que são bastante adequadas a testes empíricos.

\section{John C. Cox}

MIT Sloan School of Management

\section{Jonathan E. Ingersoll Jr.}

Yale School of Management

\section{Stephen A. Ross}

MIT Sloan School of Management

ABSTRACT This paper uses an intertemporal general equilibrium asset pricing model to study the term structure of interest rates. In this model, anticipations, risk aversion, investment alternatives, and preferences about the timing of consumption all play a role in determining bond prices. Many of the factors tradicionally mentioned as influencing the term structure are thus include in a way which is fully consistent with maximizing behavior and rational expectations. The model leads to specific formulas of bond prices which are well suited for empirical testing.

PALAVRAS-CHAVE Modelo de precificação de ativos, estrutura a termo, debêntures, teoria geral de equilíbrio, teste empírico. KEYWORDS Asset pricing model, term structure, bonds, general equilibrium theory, empirical test. 


\section{INTRODUÇÃO}

A estrutura a termo das taxas de juros mede a relação entre os rendimentos de títulos livres de inadimplência que diferem apenas em relação ao prazo até o vencimento. As determinantes dessa relação são há muito um ponto de interesse para os economistas. Ao oferecer uma tabulação completa das taxas de juros em relação ao tempo, a estrutura a termo nos fornece um meio de extrair essas informações e prever como mudanças das variáveis subjacentes afetarão a curva de rendimento.

Em um mundo feito de certezas, as taxas de juros a termo de equilíbrio devem coincidir com as taxas spot futuras; mas, quando se introduz incerteza em relação às taxas futuras, a análise se torna muito mais complexa. De modo geral, as teorias anteriores da estrutura a termo tomaram como ponto de partida o modelo de certeza e avançaram por meio do exame de generalizações estocásticas sobre as relações de equilíbrio sob condições de certeza. A literatura da área é volumosa e um levantamento abrangente seria merecedor de um artigo exclusivo. É comum, entretanto, identificar grande parte dos trabalhos anteriores como pertencentes a uma de quatro linhas de pensamento.

Primeiramente encontramos as diversas versões da hipótese das expectativas. Estas enfatizam, predominantemente, os valores esperados das taxas spot futuras ou dos retornos durante o período de manutenção. Em sua forma mais simples, a hipótese das expectativas postula que as debêntures são apreçadas de modo tal que as taxas a termo implícitas sejam iguais às taxas spot separadas. De modo geral, essa abordagem se caracteriza pelas seguintes proposições: (a) o retorno da manutenção de uma debênture até o vencimento é igual ao retorno esperado de um investimento repetido em uma série de debêntures de curto prazo, ou (b) a taxa esperada de retorno do próximo período de manutenção será a mesma para debêntures de todos os vencimentos.

A hipótese da preferência pela liquidez, proposta por Hicks (1946), concorda com a importância das taxas spot futuras esperadas, mas atribui maior peso aos efeitos das preferências dos participantes do mercado quanto ao risco. A hipótese afirma que a aversão ao risco faz com que as taxas a termo sejam sistematicamente superiores às taxas spot esperadas, geralmente em uma medida que aumenta com a data de vencimento. Esse prêmio pelo prazo é o incremento necessário para induzir os investidores a manterem títulos de mais longo prazo (mais arriscados).

Em terceiro lugar, temos a hipótese de segmentação do mercado de Culbertson (1957) e outros que oferecem uma explicação diferente dos prêmios a termo. Aqui se afirma que as pessoas têm fortes preferências quanto a vencimentos e que debêntures de diferentes vencimentos são negociadas em mercados separados e distintos. A oferta e a demanda de debêntures de um dado vencimento são, em tese, pouco afetadas pelos preços das debêntures de vencimentos "vizinhos". É claro que não há motivo, agora, para que os prêmios a termo sejam funções crescentes ou decrescentes do vencimento. Sem tentar uma crítica detalhada dessa posição, fica evidente que há um ponto em que não se pode mais sustentar que debêntures com vencimentos próximos não sejam substitutos aproximados. A possibilidade de substituição é uma parte importante da teoria que desenvolvemos.

Em sua teoria do hábitat preferido, Modigliani e Sutch (1966) usam alguns argumentos semelhantes aos da teoria da segmentação dos mercados. Mas reconhecem suas limitações e a combinam com aspectos das outras teorias. Conceberam sua abordagem como um raciocínio plausível para explicar os prêmios pelo prazo que não os restringe em termos de sinal ou monotonicidade em vez de como explicação causal necessária. ${ }^{1}$

Apesar de o foco dessas análises modernas e ecléticas da estrutura a termo sobre a explicação e teste dos prêmios pelo prazo ser desejável, esta abordagem traz duas dificuldades. Primeira, precisamos de um melhor entendimento das determinantes dos prêmios pelo prazo. As teorias anteriores são basicamente meras hipóteses que não fazem mais do que afirmar que as taxas a termo não devem ou não precisam ser iguais às taxas spot esperadas. Segunda dificuldade, todas as teorias são colocadas em termos prospectivos e precisam ser ligadas a fatos retrospectivos para serem testáveis.

As tentativas de lidar com esses dois elementos constituem a quarta linha de pesquisa sobre a estrutura a termo. Roll $(1970,1971)$, por exemplo, construiu e testou um modelo de média-variância que trata as debêntures simetricamente com outros ativos e usou uma condição de eficiência do mercado para relacionar os conceitos prospectivos e retrospectivos. ${ }^{2}$ Se a racionalidade exige que os fatos prospectivos não difiram sistematicamente do panorama retrospectivo, então podem ser realizados testes estatísticos das propostas prospectivas com base em dados retrospectivos.

Consideramos o problema da determinação da estrutura a termo como sendo um problema de teoria geral do equilíbrio, e nossa abordagem contém elementos de todas as teorias anteriores. A antecipação de eventos futuros é importante, como também o são as preferências pelo risco e as características das demais alternativas de 
investimento. Além disso, as pessoas podem ter preferências específicas quanto ao momento de seu consumo e, assim, nesse sentido, ter um hábitat preferido. Nosso modelo permite, portanto, prever detalhadamente como as mudanças ocorridas em uma ampla gama de variáveisobjeto irão afetar a estrutura a termo.

Este artigo está estruturado da seguinte maneira. A Seção 2 resume o modelo de equilíbrio desenvolvido em Cox, Ingersoll e Ross (1985) e o especializa para o estudo da estrutura a termo. Na Seção 3, derivamos e analisamos um modelo que leva a uma descrição monofatorial da estrutura a termo. A Seção 4 mostra como esse modelo pode se aplicar a outros títulos correlatos, como opções sobre debêntures. Na Seção 5 comparamos nossa abordagem pelo equilíbrio geral com uma abordagem alternativa baseada exclusivamente em arbitragem. $\mathrm{Na}$ Seção 6, consideramos alguns dos modelos mais gerais de estrutura a termo e demonstramos como os preços de mercado das debêntures podem ser usados como variáveis instrumentais em testes empíricos da teoria. A Seção 7 apresenta alguns modelos que incluem os efeitos de inflação aleatória. Na Seção 8, tecemos alguns breves comentários finais.

\section{O MODELO DE EQUILÍBRIO SUBJACENTE}

Nesta seção, revemos brevemente e especializamos o modelo de equilíbrio geral de Cox, Ingersoll e Ross (1985). O modelo é uma descrição intertemporal completa de uma economia competitiva em tempo contínuo. Lembramos que, nessa economia, há um só bem e todos os valores são medidos em termos de unidades desse bem. As oportunidades de produção consistem em um conjunto de $n$ atividades lineares. $\mathrm{O}$ vetor das taxas esperadas de retorno dessas atividades é $\alpha$ e a matriz de covariância das taxas de retorno é GG'. Os componentes $\alpha$ e $G$ são funções de um vetor $k$-dimensional de $Y$ que representa o estado da tecnologia, mudando aleatoriamente com o tempo. O desenvolvimento de $Y$ determina, assim, as oportunidades de produção que estarão disponíveis para a economia no futuro. O vetor das mudanças esperadas de $Y$ é $\mu$, e a matriz de covariância dessas mudanças é SS'.

A economia se compõe de indivíduos idênticos, cada um buscando maximizar uma função objetiva da forma

(1) $E \int_{t}^{t^{\prime}} U(C(s), Y(s), s) d s$,

onde $C(s)$ é o fluxo de consumo no tempo s, $U$ é uma curva de utilidade de Von Neumann-Morgenstern e t'é a data final. Ao realizar essa maximização, cada indivíduo escolhe seu consumo ótimo $C^{*}$, a proporção ótima $\alpha^{*}$ da riqueza $W$ a ser investida em cada um dos processos de produção e a proporção ótima $b^{*}$ da riqueza a ser investida em cada um dos direitos contingentes. Esses direitos contingentes são títulos endogenamente criados cujos payoffs são função de $W$ e $Y$. A riqueza restante a ser investida em tomada ou concessão de empréstimos à taxa de juros $r$ é, então, determinada pela restrição orçamentária. A curva indireta de utilidade $J$ é determinada pela solução do problema de maximização.

No equilíbrio desta sociedade homogênea, a taxa de juros e as taxas de retorno esperadas sobre os direitos contingentes deve se ajustar até que toda a riqueza esteja investida no processo de produção física. Esse investimento pode se dar diretamente pelos indivíduos ou indiretamente pelas empresas. Conseqüentemente, o valor de equilíbrio de $J$ é dado pela solução de um problema de planejamento em que apenas os processos de produção física estejam disponíveis. Para fins de referência futura, observamos que as condições de otimalidade para as proporções investidas terão, então, a forma

(2) $\psi \equiv \alpha W J_{W}+G G^{\prime} a^{*} W^{2} J_{W W}+G S^{\prime} W J_{W Y}-\lambda^{*} 1 \leq 0$

e $\alpha^{*} \cdot \Psi=0$, onde os subscritos de $J$ denotam derivadas parciais, JWY é um vetor ( $k \times 1)$ cujo $1^{\circ}$ elemento é JWYi', 1 é um vetor unitário ( $k \times 1)$ e $\lambda^{*}$ é um multiplicador de Lagrange. Sendo J explicitamente determinado, as condições similares de otimalidade para o problema, inclusive os direitos contingentes e a tomada e concessão de empréstimos, podem ser combinadas com as condições de fechamento do mercado para dar a taxa de juros de equilíbrio e as taxas de retorno esperado dos direitos contingentes.

Citamos, agora, dois dos principais resultados de Cox, Ingersoll e Ross (1985) de que precisaremos freqüentemente neste artigo. Primeiro, a taxa de juros de equilíbrio pode ser explicitamente escrita como

$$
\begin{aligned}
& r(W, Y, t)=\frac{\lambda^{*}}{W J_{W}} a^{* \prime} \alpha+a^{* \prime} G G^{\prime} a^{*} W\left(\frac{J_{W W}}{J_{W}}\right) \\
& +a^{* \prime} G S\left(\frac{J_{W Y}}{J_{W}}\right)=a^{* \prime} \alpha-\left(\frac{-J_{W W}}{J_{W}}\right)\left(\frac{\operatorname{var} W}{W}\right)-\sum_{i=1}^{k} \\
& \left(\frac{-J_{W Y_{\perp}}}{J_{W}}\right)\left(\frac{\operatorname{cov} W, Y_{i}}{W}\right)
\end{aligned}
$$


onde ( $\operatorname{cov} W, Y_{i}$ ) é a covariância das alterações da riqueza otimamente investida com as alterações da variável de estado $Y_{i}$, sendo $(\operatorname{var} W)$ e $\left(\operatorname{cov} Y_{i}, Y_{j}\right)$ definidas de maneira análoga; observe-se que $a^{*} \alpha$ é a taxa esperada de retorno da riqueza otimamente investida. Segundo, o valor de equilíbrio de qualquer direito contingente, $F$, deve satisfazer à equação diferencial abaixo:

$$
\text { (4) } \begin{aligned}
& \frac{1}{2} a^{* \prime} G G^{\prime} a^{*} W^{2} F_{W W}+a^{* \prime} G S^{\prime} W F_{W Y}+\frac{1}{2} \operatorname{tr}\left(S S^{\prime} F_{Y Y}\right) \\
& +\left(a^{* \prime} \alpha W-C^{*}\right) F_{W}+\mu^{\prime} F_{Y}+F_{t}+\delta-r F \\
& =\phi_{W} F_{W}+\phi_{Y} F_{Y},
\end{aligned}
$$

onde $\delta(W, Y, t)$ é o fluxo de payout recebido pelo título e

$$
\begin{aligned}
& \phi_{W}=\left(a^{* \prime} \alpha-r\right) W \\
& \phi_{Y}=\left(\frac{-J_{W W}}{J_{W}}\right) a^{* \prime} G S^{\prime} W+\left(\frac{-J_{W Y}}{J_{W}}\right)^{\prime} S S^{\prime} .
\end{aligned}
$$

Em (4), os subscritos de $F$ indicam derivadas parciais; $F_{Y}$ e $F_{W Y}$ são vetores $(k \times 1)$ e $F_{Y Y}$ é uma matriz $(k \times k)$. O lado esquerdo de (4) nos dá o rendimento excedente esperado do título acima e além do retorno livre de risco, enquanto seu lado direito nos dá o prêmio pelo risco que o título deve receber no equilíbrio. Para fins de referência futura, observamos que (4) pode ser alternativamente escrita como:

$$
\text { (6) } \begin{aligned}
& \frac{1}{2}(\operatorname{var} W) F_{W W}+\sum_{i=1}^{k}\left(\operatorname{cov} W, Y_{i}\right) F_{W Y}+\frac{1}{2} \sum_{i=1}^{k} \sum_{j=1}^{k}\left(\operatorname{cov} Y_{i}, Y_{j}\right) F_{Y_{i} Y_{j}} \\
+ & {\left[r W-C^{*}\right] F_{W}+\sum_{i=1}^{k}\left[\mu_{i}-\left(\frac{-J_{W W}}{J_{W}}\right)\left(\operatorname{cov} W, Y_{i}\right)-\sum_{j=1}^{k}\left(\frac{-J_{W Y_{j}}}{J_{W}}\right)\right.} \\
& \left.\left(\operatorname{cov} Y_{i}, Y_{j}\right)\right] F_{Y_{i}}+F_{t}-r F+\delta=0 .
\end{aligned}
$$

Para aplicar essas fórmulas ao problema da estrutura a termo das taxas de juros, especializamos a estrutura de preferências, primeiro, ao caso das curvas de utilidade de aversão relativa ao risco constante e, depois, à função logarítmica de utilidade. Mais especificamente, sejam $U(C(s), Y(s), s)$ independentes da variável de estado $Y$ e tenham eles a forma

$$
U(C(s), s)=e^{-\rho s}\left[\frac{C(s)^{\gamma}-1}{\gamma}\right],
$$

onde $\rho$ é um fator de desconto constante.
É fácil demonstrar que, nesse caso, a curva indireta de utilidade assume a forma: ${ }^{3}$

$$
J(W, Y, t)=f(Y, t) U(W, t)+g(Y, t) .
$$

Essa forma especial traz duas simplificações importantes. Primeiro, o coeficiente da aversão relativa ao risco da curva indireta de utilidade é constante, independendo das variáveis de riqueza e estado:

(9)

$$
\frac{-W J_{W W}}{J_{W}}=1-\gamma
$$

Segundo, a elasticidade da utilidade marginal da riqueza em relação a cada uma das variáveis de estado não depende da riqueza, de modo a termos

$$
\text { (10) } \frac{-J_{W Y}}{J_{W}}=\frac{-f_{Y}}{f} \text {. }
$$

Ademais, é simples verificar que as proporções ótimas $a^{*}$ da carteira dependerão de $Y$, mas não de $W$. Conseqüentemente, o vetor dos prêmios por risco de fator, $\phi_{Y}$, se reduz a $(1-\gamma) a^{* \prime} G S^{\prime}+\left(f_{Y} / f\right) S S^{\prime}$, que depende apenas de $Y$. Além disso, podemos ver em (3) que a taxa de juros de equilíbrio também depende apenas de $Y$.

A curva de utilidade logarítmica corresponde ao caso especial de $\gamma=0$. Nesse caso, é possível demonstrar que $f(Y, t)=\left[1-\exp \left[-\rho\left(t^{\prime}-t\right)\right]\right] / \rho$. Desse modo, a dependência de estado da curva indireta de utilidade só opera em $g(Y, t)$. Com isso, $\phi_{Y}$ se reduz a $\alpha^{* \prime} G S$. E a forma específica da curva indireta de utilidade nos permite resolver (2) explicitamente para $a^{*}$ como

$$
\text { (11) } a^{*}=\left(G G^{\prime}\right)^{-1} \alpha+\left(\frac{1-1\left(G G^{\prime}\right)^{-1} \alpha}{1^{\prime}\left(G G^{\prime}\right)^{-1} 1}\right)\left(G G^{\prime}\right)^{-1} 1
$$

quando todos os processos de produção estão ativos, sustentando-se uma solução análoga nos casos em que alguns processos estejam inativos.

No restante do artigo, iremos valorar títulos cujos termos contratuais não dependam explicitamente da riqueza. Como, na presença de aversão relativa ao risco constante, nem a taxa de juros $r$ nem os prêmios por fator de risco $\phi_{Y}$ dependem da riqueza, para esses títulos as derivadas parciais $F_{W}, F_{W W}$ e $F_{W Y}$ serão todas iguais a zero, e os termos correspondentes são eliminados da equação de valoração (4).

Combinando essas especializações, vemos que a equação de valoração (4) se reduz a 
(12) $\frac{1}{2} \operatorname{tr}\left(S S^{\prime} F_{Y Y}\right)+\left[\mu^{\prime}-a^{* \prime} G S^{\prime}\right] F_{Y}+F_{t}+\delta-r F=0$.

A equação (12) será a equação de valoração central neste artigo. Nós a usaremos juntamente com diversas especificações de mudança tecnológica para examinarmos a estrutura a termo implícita nas taxas de juros.

\section{UM MODELO MONOFATORIAL DA ESTRUTURA A TERMO}

Em nosso primeiro modelo da estrutura a termo das taxas de juros, admitimos que o estado da tecnologia possa ser representado por uma só variável estatística ou de estado suficiente. Esse é nosso modelo mais simples e o examinaremos em certo grau de detalhe. Isso servirá para ilustrar como uma análise similar pode ser realizada em relação aos modelos mais complexos apresentados nas seções 5 e 6 .

Premissa 1: A variação das oportunidades de produção ao longo do tempo é descrita por uma só variável de estado, $Y\left(\equiv Y_{1}\right)$.

Premissa 2: As médias e variâncias das taxas de retorno dos processos de produção são proporcionais a $\mathrm{Y}^{4}$ Assim, nem as médias nem as variâncias dominarão a decisão de carteira para grandes valores de $Y$. A variável de estado $Y$ pode ser considerada como determinante da taxa de evolução do estoque de capital no sentido descrito a seguir. Se compararmos uma situação em que $Y=\bar{Y}$, uma constante, com uma situação em que $Y=2 \bar{Y}$, então a primeira delas tem a mesma distribuição de taxas de retorno sobre um investimento fixo em qualquer processo, num prazo de dois anos, que a segunda tem num prazo de um ano. Admitimos que os elementos de $\alpha$ e $G$ sejam tais que os elementos de $a^{*}$, dados por (11), sejam positivos, de tal maneira que todos os processos estejam sempre ativos e que $1^{\prime}\left(G G^{\prime}\right)^{-1} \alpha$ seja maior que um. ${ }^{5}$

Premissa 3: O desenvolvimento da variável de estado $Y$ é dado pela equação diferencial estocástica

(13) $d Y(t)=[\xi Y+\xi] d t+v \sqrt{Y} d w(t)$,

onde $\xi e \xi$ são constantes, com $\zeta \geq 0$, e vé um vetor $1 \times(n+k)$, cujos componentes são, cada um, a constante $v_{0}$.

Essa estrutura faz com que seja conveniente introduzir a notação $\alpha=\hat{\alpha} Y, G G^{\prime} \equiv \Omega Y$ e GS' $\equiv \Sigma_{Y}$, onde os elementos $\hat{\alpha}, \Omega$ e $\Sigma$ são constantes.

Com essas premissas a respeito da mudança tecnológica e nossas premissas anteriores quanto a preferências, podemos usar (3) para escrever a taxa de juros de equilíbrio como

(14) $r(Y)=\left(\frac{1^{\prime} \Omega^{-1} \hat{\alpha}-1}{1^{\prime} \Omega^{-1} 1}\right) Y$.

A taxa de juros segue, assim, um processo de difusão com

$$
\begin{aligned}
& \text { drift } r=\left(\frac{1^{\prime} \Omega^{-1} \hat{\alpha}-1}{1^{\prime} \Omega^{-1} 1}\right)(\xi Y+\zeta) \equiv \kappa(\theta-r), \\
& \operatorname{var} r=\left(\frac{1^{\prime} \Omega^{-1} \hat{\alpha}-1}{1^{\prime} \Omega^{-1} 1}\right)^{2} v v^{\prime} Y \equiv \sigma^{2} r
\end{aligned}
$$

onde $\kappa, \theta$ e $\sigma^{2}$ são constantes, com $\kappa \theta \geq 0$ e $\sigma^{2}>0$. É conveniente definir um novo processo unidimensional de Wiener, $z_{1}(t)$, tal que:

$$
\text { (16) } \sigma \sqrt{r} d z_{1}(t) \equiv v \sqrt{Y} d w(t) \text {; }
$$

isso é admissível na medida em que cada componente de $w(t)$ é um processo de Wiener. A dinâmica da taxa de juros pode, então, ser expressa como:

(17) $d r=\kappa(\theta-r) d t+\sigma \sqrt{r} d z_{1}$.

Para $\kappa, \theta>0$, isso corresponde a um processo auto-regressivo de primeira ordem em tempo contínuo em que a taxa de juros sujeita a movimentos aleatórios é elasticamente puxada em direção a uma localização central, ou valor de longo prazo, $\theta$. O parâmetro $\kappa$ determina a velocidade do ajuste. ${ }^{6}$

Um exame do critério de classificação de fronteira demonstra que $r$ pode atingir zero se $\sigma^{2}>2 \kappa \theta$. Se $2 \kappa \theta$ $\geq \sigma^{2}$, a deriva para cima é grande o bastante para fazer com que a origem seja inacessível. ${ }^{7}$ Em qualquer dos casos, a singularidade do coeficiente de difusão na origem implica que uma taxa de juros inicialmente não negativa jamais pode vir a se tornar negativa mais adiante.

O comportamento da taxa de juros implícito nessa estrutura apresenta, assim, as seguintes propriedades empíricas relevantes: (i) Ficam excluídas taxas de juros negativas. (ii) Se a taxa de juros atingir zero, pode tornar-se positiva mais adiante. (iii) A variância absoluta da taxa de juros aumenta quando a própria taxa de juros aumenta. (iv) A taxa de juros apresenta uma distribuição de estado estável. 
A densidade probabilística da taxa de juros no momento $s$, condicionada ao seu valor no momento atual, $t$, é dada por:

$$
f(r(s), s ; r(t), t)=c e^{-u-v}\left(\frac{v}{u}\right)^{q / 2} I_{q}\left(2(u v)^{1 / 2}\right)
$$

onde:

$$
\begin{aligned}
c & \equiv \frac{2 \kappa}{\sigma^{2}\left(1-e^{-\kappa(s-t)}\right)}, \\
u & \equiv c r(t) e^{-\kappa(s-t)}, \\
v & \equiv c r(s), \\
q & \equiv \frac{2 \kappa \theta}{\sigma^{2}}-1,
\end{aligned}
$$

e Iq(.) é a curva de Bessel modificada da primeira espécie de ordem $q$. A curva de distribuição é a função qui-quadrado não central $\chi^{2}[2 c r(s) ; 2 q+2,2 u]$, com $2 q+2$ graus de liberdade e parâmetro de centralidade $2 u$ proporcional à taxa spot atual. ${ }^{8}$

Cálculos diretos dão como valor esperado e variância de $r(s)$ :

$$
\begin{aligned}
& E(r(s) \mid r(t))=r(t) e^{-\kappa(s-t)}+\theta\left(1-e^{-\kappa(s-t)}\right), \\
& \operatorname{var} .(r(s) \mid r(t))=r(t)\left(\frac{\sigma^{2}}{\kappa}\right)\left(e^{-\kappa(s-t)}-e^{-2 \kappa(s-t)}\right) \\
& +\theta\left(\frac{\sigma^{2}}{2 \kappa}\right)\left(1-e^{-\kappa(s-t)}\right)^{2} .
\end{aligned}
$$

As propriedades da distribuição das taxas de juros futuras são as esperadas. À medida que $\kappa$ se aproxima do infinito, a média vai a $\theta$ e a variância a zero, enquanto à medida que $\kappa$ se aproxima de zero, a média condicional vai à taxa de juros atual e a variância a $\sigma^{2} r(t) \cdot(s-t)$.

Se a taxa de juros apresentar reversão média $(\kappa, \theta>0)$, então, à medida que $s$ aumenta, sua distribuição se aproximará de uma distribuição gama. A curva de densidade de estado estável é:

$$
f[r(\infty), \infty ; r(t), t]=\frac{\omega^{v}}{\Gamma(v)} r^{v-1} e^{-\omega r}
$$

onde $\omega \equiv 2 \kappa / \sigma^{2}$ e $v \equiv 2 \kappa \theta / \sigma^{2}$. A média e a variância em estado estável são $\theta$ e $\sigma^{2} \theta / 2 \kappa$, respectivamente.

Tratemos, agora, do problema da valoração de uma debênture descontada livre de inadimplência que prometa pagar uma unidade no momento $T .{ }^{9}$ Os preços dessas debêntures para todos os $T$ determinarão completamente a estrutura a termo. Adotando nossas premissas, o prêmio por fator de risco em (12) é

$$
\left[\widehat{\alpha}^{\prime} \Omega^{-1} \Sigma+\left(\frac{1-1^{\prime} \Omega^{-1} \widehat{\alpha}}{1^{\prime} \Omega^{-1} 1}\right) 1^{\prime} \Omega^{-1} \Sigma\right] Y \equiv \lambda Y .
$$

Usando (15) e (21), podemos escrever de forma mais conveniente a equação fundamental do preço de uma debênture descontada, $P$ :

$$
\frac{1}{2} \sigma^{2} r P_{r r}+\kappa(\theta-r) P_{r}+P_{t}-\lambda r P_{r}-r P=0
$$

com a condição de fronteira $P(r, T, T)=1$. Os primeiros três termos de (22) são, com base na fórmula de Ito, a variação esperada do preço da debênture. Assim, a taxa esperada de retorno da debênture é $r+(\lambda r P r / P)$. O prêmio pelo retorno instantâneo de uma debênture é proporcional à elasticidade do seu juro. $\mathrm{O}$ fator $\lambda r$ é a covariância das variações da taxa de juros com as variações percentuais da riqueza otimamente investida (a "carteira de mercado"). Como $\mathrm{Pr}<0$, prêmios positivos surgirão se a covariância for negativa $(\lambda<0)$.

Podemos ver, a partir de (22), que os preços das debêntures dependem de uma só variável aleatória, a taxa de juros spot, que serve como variável instrumental para a incerteza tecnológica subjacente. Embora a sugestão de que as taxas de juros atuais (e futuras) desempenham uma função importante e, a um primeiro exame, predominante na determinação da estrutura a termo, seja genericamente aprovada, vimos que isso somente será precisamente verdadeiro sob condições especiais. ${ }^{10}$

Tomando a expectativa relevante (ver Cox, Ingersoll e Ross, 1985), obtemos os preços das debêntures como sendo:

$$
P(r, t, T)=A(t, T) e^{-B(t, T) r},
$$

onde:

$$
\begin{aligned}
& A(t, T) \equiv\left[\frac{2 \gamma e^{[(\kappa+\lambda+\gamma)(T-t)]]_{2}}}{(\gamma+\kappa+\lambda)\left(e^{\gamma(T-t)}-1\right)+2 \gamma}\right]^{2 \kappa \theta / \sigma^{2}}, \\
& B(t, T) \equiv \frac{2\left(e^{\gamma(T-t)}-1\right)}{(\gamma+\kappa+\lambda)\left(e^{\gamma(T-t)}-1\right)+2 \gamma}, \\
& \gamma \equiv\left((\kappa+\lambda)^{2}+2 \sigma^{2}\right)^{1 / 2}
\end{aligned}
$$


O preço da debênture é uma função convexa decrescente da taxa de juros e função crescente (decrescente) do tempo (vencimento). Os parâmetros do processo de taxa de juros têm os efeitos descritos a seguir. O preço da debênture é uma função convexa decrescente do nível médio da taxa de juros $\theta$ e uma função côncava crescente (convexa decrescente) da velocidade do parâmetro de ajuste $\kappa$ se a taxa de juros for maior (menor) do que $\theta$. Esses dois resultados são imediatamente evidentes a partir de seus efeitos sobre as taxas de juros esperadas futuras. Os preços das debêntures são função côncava crescente do parâmetro de risco de "mercado" $\lambda$. Intuitivamente, isso se deve principalmente ao fato de que valores mais elevados de $\lambda$ indicam maior covariância da taxa de juros com a riqueza. Assim, com um $\lambda$ maior, é mais provável que os preços das debêntures sejam maiores quando a riqueza é menor e, portanto, tem maior utilidade marginal. O preço da debênture é uma função côncava crescente da variância da taxa de juros $\sigma^{2}$. Aqui há diversos efeitos envolvidos. O mais importante é que um maior valor de $\sigma^{2}$ indica maior incerteza para com relação às oportunidades reais de produção futuras e, portanto, maior incerteza quando ao consumo futuro. Em tal mundo, os investidores avessos ao risco dariam maior valor ao direito garantido representado por uma debênture.

A dinâmica dos preços das debêntures é dada pela equação estocástica diferencial:

$$
d P=r[1-\lambda B(t, T)] P d t-B(t, T) P \sigma \sqrt{r} d z_{1}
$$

Para esse modelo de uma só variável de estado único, os retornos das debêntures têm relação perfeitamente negativa com as variações da taxa de juros. Os retornos são menos variáveis quando a taxa é menor. Com efeito, tornam-se certos se for atingida uma taxa de juros zero, já que as variações marginais da taxa de juros serão, então, certas. Como seria de se esperar intuitivamente, desde que permanecendo iguais às demais condições, a variabilidade dos retornos diminui à medida que a debênture se aproxima de seu vencimento. De fato, permitindo que $t$ se aproxime de $T$ e denotando $T$ - $t$ como $\Delta t$, vemos que a taxa esperada de retorno é $r \Delta t+O\left(\Delta t^{2}\right)$, e a variância da taxa de retorno é $O\left(\Delta t^{2}\right)$, e não $O(\Delta t)$ - como se daria no caso dos retornos do investimento nos processos produtivos ao longo de um intervalo breve. É nesse sentido que o retorno de debêntures de prazo muito curto se torna certo.

As debêntures são freqüentemente cotadas em termos de rendimentos, não de preços. Para as debêntures des- contadas de que estamos tratando, o rendimento até o vencimento, $R(r, t, T)$, é definido como $\exp [-(T-t) R(r$, $t, T)]=P(r, t, T)$. Assim, temos:

$$
R(r, t, T)=[r B(t, T)-\log \mathrm{A}(t, T)] /(T-t) .
$$

À medida que se aproxima o vencimento, o rendimento até o vencimento se aproxima da taxa de juros atual, independentemente de quaisquer dos parâmetros. Ao considerarmos vencimentos cada vez mais distantes, o rendimento se aproxima de um limite que independe da taxa de juros atual:

$$
\text { (26) } R(r, t, \infty)=\frac{2 \kappa \theta}{\gamma+\kappa+\lambda}
$$

Quando a taxa spot está abaixo desse rendimento de longo prazo, a estrutura a termo é uniformemente ascendente. Com uma taxa de juros superior a $\kappa \theta /(\kappa+$ $\lambda$ ), a estrutura a termo é descendente. Para valores intermediários da taxa de juros, a curva de rendimento é saliente (humped.)

Outras estatísticas comparativas da curva de rendimento podem ser facilmente obtidas a partir da função de precificação de debêntures. Um aumento da taxa de juros atual eleva os rendimentos de todos os vencimentos, mas o efeito é mais pronunciado para maiores vencimentos. Da mesma forma, um aumento do estado estável significa que $\theta$ aumenta para todos os rendimentos, mas, aqui, o efeito é maior para vencimentos mais distantes. Os rendimentos até o vencimento diminuem com o aumento de $\sigma^{2}$ ou de $\lambda$, enquanto o efeito de uma variação de $\kappa$ pode ter qualquer sinal, dependendo da taxa de juros atual.

Sempre houve considerável preocupação com as previsões sem viés das taxas de juros futuras. $\mathrm{Na}$ atual situação, podemos trabalhar diretamente com a equação (19), que dá os valores esperados das taxas de juros futuras em termos da taxa atual e dos parâmetros $\kappa$ e $\theta$. Mas, no modelo de expectativas racionais que construímos, todas as informações atualmente conhecidas sobre o movimento futuro das taxas de juros se encontra nos preços atuais das debêntures e na estrutura a termo. Se o modelo estiver correto, qualquer parâmetro poderá ser determinado a partir da estrutura a termo e dos valores dos demais parâmetros.

Essa abordagem é especialmente importante quando o modelo é ampliado para permitir um termo de deriva dependente do tempo, $\theta(t)$. Com isso, podemos usar as 
informações contidas na estrutura a termo para obter $\theta(t)$ e as taxas spot futuras esperadas sem precisar impor restrições anteriores à sua forma funcional.

Agora a taxa spot futura esperada dada por (19) é alterada para:

$$
E(r(T) \mid r(t))=r(t) e^{-\kappa(T-t)}+\kappa \int_{t}^{T} \theta(s) e^{-\kappa(T-s)} d s
$$

A fórmula de precificação de debêntures (30), por sua vez, é modificada para:

$$
P(r, t, T)=\widehat{A}(t, T) e^{-B(t, T) r}
$$

onde

(29) $\widehat{A}(t, T)=\exp \left(-\kappa \int_{t}^{T} \theta(s) B(s, T) d s\right)$,

o que se reduz a (23) quando $\theta($ s) é constante.

Admitindo, para exemplificar, que sejam conhecidos os demais parâmetros do processo, podemos usar a estrutura a termo para determinar previsões sem viés das taxas de juros futuras. Por meio de (28), Â( $t, T)$ é função observável de $T$, dado a estrutura a termo e a forma conhecida de $\mathrm{B}(t, T)$, e técnicas padronizadas podem ser usadas para inverter (29) e obter uma expressão de $\theta(t)$ em termos de $\hat{A}(t, T)$ e $B(t, T)$. A equação (27) pode agora ser usada para se obterem previsões dos valores esperados das taxas spot futuras implícitas na atual estrutura a termo.

Observe que esses não são os mesmos valores que seriam dados pela premissa tradicional quanto às expectativas de que os valores esperados das taxas spot futuras estão contidos na estrutura a termo sob a forma de taxas a termo implícitas. Em um modelo de tempo contínuo, a taxa a termo $r(T)$ é dada por $-P_{T} / P$. Então, diferenciando (28):

$$
\text { (30) } \begin{aligned}
& \hat{r}(T)=-P_{T}(r, t, T) / P(r, t, T) \\
& =r B_{T}(t, T)+\kappa \int_{t}^{T} \theta(s) B_{T}(s, T) d s .
\end{aligned}
$$

Comparando (27) e (30), vemos que ambas têm a mesma forma geral. Mas a preditiva tradicional da taxa a termo aplica indevidamente os pesos $B_{T}(s, T) \neq e^{-\kappa(T-s)}$, resultando em uma previsão enviesada.

Diversas especificações alternativas da dependência temporal também podem ser incluídas com pequenas alterações no modelo. Um exemplo especialmente interessante leva a uma taxa de juros de $\bar{r}(t)+g(t)$, onde $\bar{r}(t)$ é dado por (17) e $g(t)$ é uma função que fornece uma fronteira inferior positiva para a taxa de juros. $\mathrm{O}$ essencial em todos esses casos é que, no modelo das expectativas racionais, a atual estrutura a termo incorpore as informações necessárias para avaliar a distribuição probabilística do mercado da rota futura das taxas de juros. Além disso, a estrutura a termo pode ser invertida para identificar essas especificações.

Outras especificações univariadas da mudança tecnológica implicarão, por sua vez, outras propriedades estocásticas da taxa de juros. É fácil verificar que, em nosso modelo, se $\alpha$ e $G G$ ' forem proporcionais a uma função $h(Y, t)$, então a taxa de juros também será proporcional a $h(Y, t)$. Por meio de uma escolha adequada de $h(Y, t), \mu(Y, t)$ e $S(Y, t)$ é possível incluir uma ampla gama de propriedades prospectivas de movimento das taxas de juros dentro do contexto de um modelo completamente consistente.

\section{VALORAÇÃO DE ATIVOS COM PAYOFFS DEPENDENTES DA TAXA DE JUROS GERAL}

Nosso arcabouço de valoração pode ser facilmente aplicado a outros títulos, cujos payoffs dependam das taxas de juros, como opções de debêntures e futuros de debêntures. Essa flexibilidade permite que o modelo forneça previsões sobre os padrões de precificação que devem prevalecer simultaneamente em diferentes mercados financeiros. Conseqüentemente, aplicações a outros títulos podem permitir testes empíricos mais ricos e poderosos do que os possíveis apenas com o mercado de debêntures.

Como exemplo da valoração de outros tipos de títulos onerosos, vamos considerar as opções de debêntures. Denotemos como $\mathrm{C}(\mathrm{r}, t, T, \mathrm{~s}, K)$ o valor no momento $t$ de uma opção call sobre uma debênture descontada com data de maturidade $s$, preço de exercício $K$ e data de vencimento $T .{ }^{11} \mathrm{O}$ preço da opção seguirá a equação básica de valoração com a condição terminal:

(31) $\mathrm{C}(\mathrm{r}, t, T ; \mathrm{s}, \mathrm{K})=\max [\mathrm{P}(\mathrm{r}, T, \mathrm{~s})-K, 0]$.

Fica entendido que $S \geq T \geq t$ e que $K$ fica restrito a ser menor que $A(t, s)$, o máximo preço possível da debênture no momento $T$, já que, do contrário, a opção jamais seria exercida e não teria valor. Tomando novamente as expectativas relevantes, chegamos à seguinte fórmula do 
preço da opção:

(32)

$$
\begin{aligned}
& \mathrm{C}(\mathrm{r}, t, T, \mathrm{~s}, K) \\
& =P(r, t, s) \chi^{2}\left(2 r *[\phi+\psi+B(T, s)] ; \frac{4 \kappa \theta}{\sigma^{2}}, \frac{2 \phi^{2} r e^{\gamma}(T-t)}{\phi+\psi+B(T, s)}\right) \\
& -K P(r, t, T) \chi^{2}\left(2 r *[\phi+\psi] ; \frac{4 \kappa \theta}{\sigma^{2}}, \frac{2 \phi^{2} r e^{\gamma}(T-t)}{\phi+\psi}\right),
\end{aligned}
$$

onde

$$
\begin{aligned}
& \gamma \equiv\left((\kappa+\lambda)^{2}+2 \sigma^{2}\right)^{1 / 2}, \\
& \phi \equiv \frac{2 \gamma}{\sigma^{2}\left(e^{\gamma(T-t)}-1\right)}, \\
& \psi \equiv(\kappa+\lambda+\gamma) / \sigma^{2}, \\
& r^{*} \equiv\left[\log \left(\frac{A(T, s)}{K}\right)\right] / B(T, s),
\end{aligned}
$$

e $\chi^{2}(\cdot)$ é a função de distribuição qui-quadrado não central anteriormente apresentada; $r^{*}$ é a taxa de juros crítica abaixo da qual o exercício irá ocorrer, ou seja, $\mathrm{K}=\mathrm{P}\left(r^{*}, T, \mathrm{~s}\right)$.

A opção call é função crescente do vencimento (mantida fixa a data de expiração em que vence a debênture objeto). Opções call sobre ações é uma função crescente da taxa de juros, em parte porque um aumento das taxa reduz o valor presente do preço de exercício. Aqui, contudo, um aumento da taxa de juros também irá deprimir o preço da debênture objeto. Uma análise numérica indica que esse último efeito é mais forte e que o valor da opção é uma função convexa decrescente da taxa de juros. As demais estatísticas comparativas são indeterminadas.

\section{COMPARAÇÃO COM OS MÉTODOS DE PRECIFICAÇÃO DE DEBÊNTURES POR ARBITRAGEM}

Nesta seção, faremos uma breve comparação de nossa metodologia com alguns meios alternativos de modelagem da precificação de debêntures em tempo contínuo. É útil que isso seja feito agora, e não mais adiante, porque o modelo da Seção 3 fornece um padrão ideal de comparação.

Nossa abordagem parte de uma descrição detalhada da economia subjacente. Isso nos permite especificar os seguintes ingredientes da precificação de debêntures: (a) as variáveis de que depende o preço da debênture; (b) as propriedades estocásticas das variáveis objeto que são endogenamente determinadas; e (c) a forma exata dos prêmios por fator de risco. Merton (1970) demonstra que, quando se parte da imposição direta de premissas quanto a (a) e (b), a fórmula de Ito pode ser usada para declarar o rendimento excedente esperado de uma debênture como a forma à esquerda de (4). Se a forma funcional do lado direito de (4) fosse conhecida, seria possível obter uma equação de precificação de debêntures. Por exemplo, admitindo, arbitrariamente, que os preços das debêntures dependam exclusivamente da taxa de juros spot, $r$, que a taxa de juros siga o processo dado em (17) e que o rendimento excedente esperado de uma debênture com data de vencimento $T$ seja $Y(\mathrm{r}, \mathrm{s}, T)$, obter-se-ia

(33) $\frac{1}{2} \sigma^{2} r P_{r r}+\kappa(\theta-r) P_{r}+P_{t}-r P=\mathrm{Y}(r, t, T)$.

E se há algum equilíbrio subjacente que sustente as premissas (a) e (b), deve haver alguma função de $Y$ para a qual os preços das debêntures sejam dados por (33). Como observa Merton, contudo, essa derivação em si não fornece qualquer meio de determinar $Y$ ou relacioná-lo com as variáveis objeto reais.

Uma abordagem à precificação de debêntures por arbitragem foi desenvolvida numa série de estudos por Brennan e Schwartz (1979), Dothan (1978), Garman (1977), Richard (1978) e Vasicek (1977). Argumentos semelhantes aos usados na prova do Teorema 2 de Cox, Ingersoll e Ross (1985) são também usados para demonstrar que, na ausência de oportunidades de arbitragem, $Y$ deve ter a forma

(34) $\mathrm{Y}(r, t, T)=\psi(r, t) P_{r}(r, t, T)$,

onde $\Psi$ é uma função que depende apenas do tempo-calendário e não da data de vencimento da debênture. Isso impõe restrições definidas à forma do rendimento excedente esperado; nem todas as funções de $Y$ satisfarão tanto (33) quanto (34).

Há, entretanto, alguns problemas em potencial em se dar o passo seguinte e usar a abordagem por arbitragem para determinar um modelo completo e específico da estrutura a termo. A abordagem em si não oferece nenhum meio de garantir que haja algum equilíbrio subjacente para o qual as premissas (a) e (b) sejam consistentes. Deixando de lado esse problema, outra dificuldade surge do fato de que a abordagem por arbitragem não implica que todas as escolhas de $\Psi$ em (34) levem a preços de debêntures que não admitam oportunidades de arbitra- 
gem. De fato, fechar o modelo assumindo uma forma funcional específica de $\Psi$ pode levar a inconsistências internas.

Como exemplo do problema em potencial, consideremos (33) com Y como consta em (34). Isso nos dá a equação de valoração

(35) $\frac{1}{2} \sigma^{2} r P_{r r}+\kappa(\theta-r) P_{r}+P_{t}-r P=\psi(r, t) P_{r}$,

idêntica a (22) a não ser por uma especificação da função $\Psi$. Poderíamos, agora, fechar o modelo admitindo que $\Psi$ seja linear à altura da taxa spot, $\Psi(r, t)=\Psi_{0}+\lambda r$.

A solução de (35) será, então

(36) $P(r, t, T)=[A(t, T)]^{\left(\kappa \theta-\psi_{0}\right) / \kappa \theta} \exp [-r B(t, T)]$,

e o comportamento dinâmico do preço da debênture será dado por

(37) $d P=\left[r-\left(\psi_{0}+\lambda r\right) B(t, T)\right] P d t-B(t, T) \sigma \sqrt{r} P d z_{1}$.

A forma linear admitida para o prêmio pelo risco parece bastante razoável e aparentaria ser uma boa opção para trabalhos empíricos, mas, na verdade, produz um modelo instável. Isso pode ser percebido com mais facilidade quando $r=0$. Nesse caso, o retorno da debênture no instante seguinte seria livre de risco; ainda assim, seu preço apreciaria à taxa - $\Psi_{0} B(t, T)$, diferente da taxa de juros zero em vigor. ${ }^{12}$ Temos, assim, um modelo que garante oportunidades de arbitragem em vez de as excluir. A dificuldade, evidentemente, está em não haver um equilíbrio subjacente que possa dar sustentação aos prêmios presumidos.

A abordagem pelo equilíbrio aqui desenvolvida tem, portanto, duas vantagens importantes em relação aos métodos alternativos de valoração de debêntures em tempo contínuo. Primeiro, garante automaticamente que o modelo possa ser completamente especificado sem perda de consistência interna. Segundo, fornece um meio de prever como mudanças das variáveis econômicas reais afetarão a estrutura a termo.

\section{MODELOS MULTIFATORIAIS DE ESTRUTURA A TERMO E $O$ USO DE PREÇOS COMO VARIÁVEIS INSTRUMENTAIS}

Na Seção 3, especializamos o arcabouço de equilíbrio geral de Cox, Ingersoll e Ross (1985) para desenvolver um modelo completo de precificação de debêntures.
Escolhemos, intencionalmente, uma especificação simples para ilustrar as informações detalhadas que tal modelo é capaz de produzir. No modelo, os preços das debêntures de todos os vencimentos dependem de um só fator explicativo aleatório, a taxa de juros spot. Embora a estrutura a termo resultante assuma diversas formas alternativas, é inerente a um modelo monofatorial que as mudanças dos preços das debêntures de todos os vencimentos apresentem perfeita correlação. Tal modelo também implica que os preços das debêntures não dependem da rota que a taxa de juros seguir para atingir seu nível atual. Para algumas aplicações, essas propriedades podem ser por demais restritivas. Contudo, especificações mais genéricas das oportunidades tecnológicas implicarão, por sua vez, modelos de precificação mais genéricos. As estruturas multifatoriais resultantes terão mais flexibilidade do que o modelo monofatorial, mas também serão, inevitavelmente, de análise mais trabalhosa e difícil.

Para ilustrar as possibilidades, consideremos duas generalizações simples de nosso modelo anterior. Suponhamos que, em nossa descrição da mudança tecnológica, em (13) e (15), se permita que o próprio parâmetro central de tendência $\theta$ varie aleatoriamente com a equação

(38) $d \theta=v(Y-\theta) d t$,

onde $v$ é uma constante positiva. Em outras palavras, sejam $\theta \equiv Y_{2}$ e $\mu_{2}=v\left(Y_{1}-Y_{2}\right)$. O valor de $\theta$ a qualquer tempo será, portanto, uma integral exponencialmente ponderada dos valores passados de I. Pode-se verificar, então, que a taxa de juros $r$ é dada novamente por (14), e que o preço da debênture $P$ terá a forma

(39) $P(r, \theta, t, T)=\exp [-r f(t, T)-\theta g(t, T)]$,

onde $f$ e $g$ são funções explicitamente determináveis do tempo. Nesse caso, tanto o rendimento até o vencimento das debêntures descontadas, como os valores esperados das taxas de juros spot futuras, serão funções lineares das taxas spot atual e passadas. ${ }^{13}$

Como segunda generalização, suponhamos que os coeficientes de produção $\alpha$ e GG' sejam proporcionais à soma das duas variáveis aleatórias independentes $Y_{1}$ e $Y_{2}$, cada uma seguindo uma equação da forma (13). Pode-se demonstrar, então, que a taxa de juros spot $r$ será proporcional à soma de $Y_{1}$ e $Y_{2}$, e que os preços das debêntures terão, novamente, a foram exponencial

(40) $P\left(r, Y_{2}, t, T\right)=f(t, T) \exp \left[-r g(t, T)-Y_{2} h(t, T)\right]$, 
onde $f, g$ e $h$ são outras funções explicitamente determinadas do tempo. Nesse modelo, as variações dos preços das debêntures de todos os vencimentos já não estão mais perfeitamente correlacionadas.

Cada uma dessas generalizações leva a um modelo bifatorial da estrutura a termo e as curvas de rendimento resultantes podem assumir uma ampla variedade de formatos. Maiores generalizações multifatoriais podem ser construídas de forma semelhante.

Em cada um dos modelos aqui considerados, uma variável explicativa não é diretamente observável. Generalizações multifatoriais tenderão a herdar de forma ainda mais grave esse problema. Conseqüentemente, em aplicações empíricas pode ser conveniente usar alguns dos preços endogenamente determinados como variáveis instrumentais para eliminar as variáveis que não possam ser diretamente observadas. Em alguns casos, isso será possível. Escolhamos como variáveis instrumentais a taxa de juros spot, $r$, e um vetor das taxas de juros de longo prazo, l. Em geral, cada uma dessas taxas de juros será função de $W$ (a menos que a curva de utilidade comum seja isoelástica) e de todas as variáveis de estado. Se for possível inverter esse sistema globalmente e expressar essas últimas como funções duplamente diferenciáveis de $r$ e l, então $r$ e $l$ poderão ser usadas como variáveis instrumentais de maneira condizente com o arcabouço de equilíbrio geral.

Para fins exemplificativos, suponhamos haver duas variáveis de estado, $Y_{1}$ e $Y_{2}$, e que a utilidade seja isoelástica de tal modo que o nível de riqueza seja irrelevante. Então, para as variáveis instrumentais $r$ e $l$, uma escalar, o uso de cálculos diretos, embora complexos, demonstra que a equação de valoração (4) pode ser reescrita como:

$$
\text { (41) } \begin{aligned}
& \frac{1}{2}(\operatorname{var} r) F_{r r}+(\operatorname{cov} r, l) F_{r l}+\frac{1}{2}(\operatorname{var} l) F_{l l}+\left[\mu_{r}-\lambda_{r}(r . l)\right] F_{r} \\
& +\left[\mu_{l}-\lambda_{l}(r . l)\right] F_{l}-r F+F_{l}+\delta=0 .
\end{aligned}
$$

As funções $\lambda_{r}$ e $\lambda_{l}$ representam o papel de prêmios por fator de risco em (5). Estão relacionadas aos prêmios por fator de risco, $\emptyset_{Y}$, por meio de:

$$
\begin{aligned}
& \lambda_{r}(r, l)=\left[\psi_{1} \frac{\partial g}{\partial l}-\psi_{2} \frac{\partial f}{\partial l}\right] / \Delta, \\
& \lambda_{l}(r, l)=\left[\psi_{2} \frac{\partial f}{\partial r}-\psi_{1} \frac{\partial g}{\partial r}\right] / \Delta,
\end{aligned}
$$

onde
(42) $Y_{1} \equiv f(r, l, t), \quad Y_{2} \equiv g(r, l, t)$

$$
\begin{aligned}
& \phi_{Y_{1}}\left(Y_{1}, Y_{2}, t\right) \equiv \psi_{1}(r, l, t), \quad \phi_{Y_{2}}\left(Y_{1}, Y_{2}, t\right) \equiv \psi_{2}(r, l, t), \\
& \Delta \equiv \frac{\partial f}{\partial r} \frac{\partial g}{\partial l}-\frac{\partial f}{\partial l} \frac{\partial g}{\partial r} .
\end{aligned}
$$

Até aqui, não usamos o fato de que l é taxa de juros e que a transformação de (4) em (41) pode ser realizada para uma variável instrumental arbitrária se a inversão for possível. A vantagem de escolher uma instrumental que seja taxa de juros está em que o segundo prêmio por fator de risco, $\lambda_{l}$, e a deriva, $\mu_{l}$, podem ser eliminados de (41), como vemos a seguir.

Seja $Q$ o valor específico de uma debênture cujo rendimento continuamente composto até o vencimento seja $l$. Denote-se por $\mathrm{c}(t)$ o fluxo de pagamentos da debênture, inclusive cupons e retorno sobre o principal. De modo geral, esse fluxo será zero durante a maior parte do tempo, com impulsos representando uma taxa infinita de fluxo sempre que se fizrem pagamentos. Como, por definição, $Q \equiv \int_{t}^{T} c(s) \exp [-l(s-t)] d s$, podemos escrever:

$$
\begin{aligned}
& Q \equiv \Lambda_{0}(l), \quad Q_{l} \equiv \Lambda_{1}(l) \\
& Q_{l l} \equiv \Lambda_{2}(l), \quad Q_{t} \equiv-c(t)+l \Lambda_{0}(l)=-\delta+l \Lambda_{0}(l), \\
& Q_{r}=Q_{r r}=Q_{r l}=0
\end{aligned}
$$

onde

$$
\Lambda_{\mathrm{n}} \equiv \int_{t}^{T}(t-s)^{n} c(s) e^{-1(s-t)} d s
$$

e a integral deve ser interpretada no sentido dado em Stieltjes. Substituindo (43) em (41), temos

$$
\mu_{l}-\lambda_{l}(r, l)=\frac{(r-l) \Lambda_{0}(l)-\frac{1}{2}(\operatorname{var} l) \Lambda_{2}(l)}{\Lambda_{1}(l)}
$$

e o prêmio pelo fator de risco não observável pode ser substituído pela função observável de (44). Se Q for um console bond com cupons pagos continuamente à taxa $c$, então $\Lambda_{0}=c / l, \Lambda_{1}=-c / l^{2}, \Lambda^{2}=2 c / l^{3}$ e (44) pode ser escrita como: ${ }^{14}$

$$
\mu_{l}-\lambda_{l}(r, l)=\frac{(\operatorname{var} l)}{l}+l(l-r)
$$

Essas representações podem ser um ponto de partida 
útil para trabalhos empíricos. Mas é importante lembrar que não podem ser plenamente justificadas sem considerar as características da economia. Na próxima seção examinaremos mais alguns modelos de múltiplas variáveis de estado, todos os quais podem ser reexpressos sob essa forma.

\section{INCERTEZA INFLACIONÁRIA E A PRECIFICAÇÃO DE DEBÊNTURES NOMINAIS}

O modelo aqui apresentado lida com uma economia real em que a moeda não teria utilidade. Para acrescentar um papel válido para a moeda, teríamos que introduzir características adicionais que nos distanciariam de nosso objetivo original. Mas, em um mundo em que alterações da oferta de moeda não têm efeitos reais, podemos introduzir artificialmente alguns aspectos da moeda e da inflação, imaginando que uma das variáveis de estado represente um nível de preços e que alguns contratos tenham payoffs cujo valor real dependa desse nível de preços. Ou seja, encontram-se especificadas em temos nominais. Nada disso exige alterações à teoria em geral.

Seja o nível de preços, $p, a k^{a}$ variável de estado. Como admitimos que essa variável não tem efeito sobre o equilíbrio real subjacente, as funções $\alpha, \mu, G, S$, e J independem de $p$. É claro que isso não impediria que mudanças de $p$ estivessem estatisticamente correlacionadas com mudanças da riqueza real e outras variáveis de estado. Sob tais circunstâncias, o valor real de um direito cujo payoff esteja denominado em termos nominais ainda atenderá à equação (4). Só o que resta fazer é expressar o payoff nominal em termos reais para as condições de fronteira. Alternativamente, a equação de valoração (4) também se sustentará se $p$ for uma função diferenciável de $W, Y$ e $t .{ }^{15}$

Podemos ilustrar alguns desses pontos no contexto do modelo da Seção 3. Tomemos uma segunda variável de estado para que seja o nível de preços, $p\left(\equiv Y_{2}\right)$, e consideremos como valorar um contrato que pagará certamente no momento $T$ um montante $1 / p(T)$. Suponhamos que o nível de preços $p$ se mova de acordo com

(46) $d p=\mu(p) d t+\sigma(p) d w_{\mathrm{n}+2}(t)$

e que isso não esteja correlacionado com $W$ e $Y_{1}$. Admitamos ainda que os coeficientes de (45) sejam tais que $E[p-1(s)]$ existam para todos os valores finitos de $s$.
Teríamos, então, a equação de valoração de $N$

$$
\begin{aligned}
& \frac{1}{2} \sigma^{2} r N_{r r}+\frac{1}{2} \sigma^{2}(p) N_{p p}+[\kappa \theta-(\kappa+\lambda) r] N_{r} \\
& +\mu(p) N_{p}+N_{t}-r N=0
\end{aligned}
$$

com a condição terminal $N(r, p, T, T)=1 / p(T)$. É possível verificar diretamente que a solução é

$$
\text { (48) } N(r, p, t, T)=P(r, t, T) \underset{p(t), t}{E}[1 / p(T)]
$$

onde $P$ é o preço de uma debênture descontada real dado em (23).

Nessa formulação, a taxa esperada de inflação só muda com o nível de preços. Para o caso freqüentemente admitido de preços de distribuição logarítmica normal, contudo, $\mu(p)=\mu_{p} p, \sigma(p)=\sigma_{p} p, \mathrm{e}$

(49) $N(r, p, t, T)=e^{-\left(\mu_{p}-\sigma_{p}^{2}\right)(T-t)} P(r, t, T) / p(t)$,

de tal modo que, nesse caso, o preço de uma debênture nominal em termos nominais, $\widehat{N} \equiv p(t) N$, seria independente do atual nível de preços. Com os preços em distribuição logarítmica normal, a taxa esperada de inflação é constante, embora, evidentemente, a inflação efetiva não o seja.

Em um exemplo mais genérico, podemos separar a taxa esperada de inflação do fator de nível de preços e identificá-la com uma terceira variável de estado. Mais uma vez, não será necessário alterar a teoria. Chamemos a taxa esperada de inflação de $y$. Propomos dois modelos alternativos do comportamento da taxa de inflação: (i) Modelo 1,

$$
d y=\kappa_{1} y\left(\theta_{1}-y_{1}\right) d t+\sigma_{l} y^{3 / 2} d z_{3}
$$

(ii) Modelo 2,

(51) $d y=\kappa_{2}\left(\theta_{2}-y\right) d t+\sigma_{2} y^{1 / 2} d z_{3}$

sendo que a equação diferencial estocástica que rege o movimento do nível de preços em cada caso

$$
\text { (52) } d p=y p d t+\sigma_{p} p y^{1 / 2} d z_{2}
$$

$\operatorname{com}(\operatorname{cov} y, p) \equiv \rho \sigma_{1} \sigma_{p} y^{2} p$ no Modelo $1,(\operatorname{cov} y, p) \equiv \rho \sigma_{1} \sigma_{p} y p$ no Modelo 2 e $\sigma_{p}<1$.

Aqui, como em (17), definimos, por questão de conveniência, $z_{2}(t)$ e $z_{3}(t)$ como as combinações lineares adequadas de $w_{n+2}(t)$ e $w_{n+3}(t)$. 
O Modelo 1 pode ser a melhor escolha empírica, já que evidências informais sugerem que a variância relativa (percentual) da taxa esperada de inflação aumenta com o nível da inflação. O Modelo 1 traz essa propriedade, ao contrário do Modelo 2. Mas a solução do Modelo 2 é mais tratável, de modo que registraremos os dois para possível uso empírico. Nos dois modelos a taxa esperada de inflação é trazida para um nível de equilíbrio de longo prazo. Os dois modelos também permitem correlação entre as variações do nível de inflação e as variações do nível de preços, admitindo, assim, forças extrapolativas positivas ou negativas no movimento do nível de preços.

A equação de valoração para o valor real de uma debênture nominal, especializada para nosso exemplo com o Modelo 1, será, portanto,

$$
\text { (53) } \begin{aligned}
& \frac{1}{2} \sigma^{2} r N_{r r}+\frac{1}{2} \sigma_{1}^{2} y^{3} N_{y y}+\rho \sigma_{1} \sigma_{p} y^{2} p N_{y p}+\frac{1}{2} \sigma_{p}^{2} p^{2} y N_{p p} \\
& +[\kappa \theta-(\kappa+y) r] N_{r}+\kappa_{1} y\left(\theta_{1}-y\right) N_{y}+y p N_{p}+ \\
& N_{t}-r N=0
\end{aligned}
$$

sendo $N(r, y, p, T, T)=1 / p(T)$. A solução da equação (53) é

$$
N(r, y, p, t, T)=\frac{\Gamma(v-\delta)}{\Gamma(v)}\left[\frac{c(t)}{y}\right]^{\delta} M\left(\delta, v,-\frac{c(t)}{y}\right) P(r, t, T) / p(t),
$$

onde

$$
\text { (54) } \begin{aligned}
c(t) & \equiv \frac{2 \kappa_{1} \theta_{1}}{\sigma_{1}^{2}\left(e^{\kappa_{1} \theta_{1}(T-t)}-1\right)}, \\
\delta & \equiv\left[\left[\left(\kappa_{1}=\rho \sigma_{1} \sigma_{p}+\frac{1}{2} \sigma_{1}^{2}\right)^{2}+2\left(1-\sigma_{p}^{2}\right) \sigma_{1}^{2}\right]^{1 / 2}\right. \\
- & \left.\left(\kappa_{1}+\rho \sigma_{1} \sigma_{p}+\frac{1}{2} \sigma_{1}^{2}\right)\right] / \sigma_{1}^{2}, \\
v & \equiv 2\left[(1+\delta) \sigma_{1}^{2}+\kappa_{1}+\rho \sigma_{1} \sigma_{p}\right] / \sigma_{1}^{2},
\end{aligned}
$$

$M(\cdot, \cdot, \cdot)$ é a função hipergeométrica confluente e $\Gamma(\cdot)$ é a função gama. ${ }^{16}$

Procedendo da mesma forma com o Modelo 2, obtemos a equação de valoração:

(55) $\frac{1}{2} \sigma^{2} r N_{r r}+\frac{1}{2} \sigma_{2}^{2} y N_{y y}+\rho \sigma_{2} \sigma_{p} y p N_{y p}+\frac{1}{2} \sigma_{p}^{2} y p^{2} N_{p p}$

$$
+[\kappa \theta-(\kappa+\lambda) r] N_{r}+\kappa_{2}\left[\theta_{2}-y\right] N_{y}+y p N_{p}+N_{t}-r N=0
$$

onde $N(r, y, p, T, T)=1 / p(T)$. A fórmula de valoração correspondente é:

$$
\begin{aligned}
& N(r, y, p, t, T) \\
(56) & =\left(\frac{2 \xi e^{\left[\left(\kappa_{2}+\rho \sigma_{2} \sigma_{p}+\xi\right)(T-t)\right] / 2}}{\left(\xi+\kappa_{2}+\rho \sigma_{2} \sigma_{p}\right)\left(e^{\xi(T-t)}-1\right)+2 \xi}\right)^{2 \kappa_{2} \theta_{2} / \sigma_{2}^{2}} \\
& \times \exp \left(\frac{-2\left(e^{\xi(T-t)}-1\right)\left(1-\sigma_{p}^{2}\right) y}{\left(\xi+\kappa_{2}+\rho \sigma_{2} \sigma_{p}\right)\left(e^{\xi(T-t)}-1\right)+2 \xi}\right) P(r, t, T) / p(t),
\end{aligned}
$$

onde

$$
\xi \equiv\left[\left(\kappa_{2}+\rho \sigma_{2} \sigma_{p}\right)^{2}+2 \sigma_{2}^{2}\left(1-\sigma_{p}^{2}\right)\right]^{1 / 2} .
$$

A estrutura a termo das taxas de juros implícitas em (54) e (56) pode assumir uma ampla variedade de formatos, dependendo dos valores relativos das variáveis e parâmetros. Modelos mais complexos com efeitos mais detalhados podem ser construídos de forma semelhante.

Em todo este estudo, usamos especializações da equação fundamental de valoração (6). Essa equação determina o valor real de um direito contingente como função da riqueza real e das variáveis de estado. Para alguns fins empíricos, pode ser conveniente dispor de uma equação de valoração correspondente em que todos os valores sejam expressos em termos nominais.

Em nosso cenário, isso é dado pela seguinte proposição. Seja a riqueza nominal $X \equiv p W$, seja a curva de utilidade indireta em termos da riqueza nominal $V(X, Y, t) \equiv J(X / p, Y, t) \equiv J(W, Y, t)$, e seja o valor nominal de um direito em termos da riqueza nominal $H(X, Y, t)$ $\equiv p F(X / p, T, t) \equiv p F(W, Y, t)$. Como antes, seja $p$ o $k^{o}$ elemento de $Y$.

Proposição: $O$ valor nominal de um direito contingente em termos da riqueza nominal, $H(X, Y, t)$, satisfaz a equação diferencial parcial

$$
\begin{aligned}
& \frac{1}{2}(\operatorname{var} X) H_{X X}+\sum_{i=1}^{k}\left(\operatorname{cov} X, Y_{i}\right) H_{X Y_{i}}+\frac{1}{2} \sum_{i=1}^{k} \sum_{j=1}^{k}\left(\operatorname{cov} Y_{i}, Y_{j}\right) H_{Y_{i} Y_{j}} \\
(57)+ & +\left(\iota X-p C^{*}\right) H_{\chi}+\sum_{i=1}^{k}\left[\mu_{i}-\left(\frac{-V_{X X}}{V_{X}}\right)\left(\operatorname{cov} X, Y_{i}\right)\right. \\
& \left.-\sum_{j=1}^{k}\left(\frac{-V_{X Y_{j}}}{V_{X}}\right)\left(\operatorname{cov} Y_{i}, Y_{j}\right)\right] H_{Y_{i}}+H_{t}+p \delta-\iota H=0,
\end{aligned}
$$

onde a taxa de juros nominal, $\iota$, é dada por 
(58) $\iota=\alpha_{x}-\left(\frac{-V_{X Y}}{V_{X}}\right)\left(\frac{\operatorname{var} X}{X}\right)-\sum_{i=1}^{k}\left(\frac{-V_{X Y_{i}}}{V_{X}}\right)\left(\frac{\operatorname{cov} X, Y_{i}}{X}\right)$

e ax é a taxa esperada de retorno sobre a riqueza nominal,

$$
\alpha_{x}=\alpha^{* \prime} \alpha+\left(\frac{\mu_{p}}{p}\right)+\left(\frac{\operatorname{cov} p, X}{p X}\right)-\left(\frac{\operatorname{var} p}{p^{2}}\right) .
$$

Prova: A regra de multiplicação de Ito implica que $(\operatorname{var} W)=\left(1 / p^{2}\right)(\operatorname{var} X)-\left(2 X / p^{3}\right)(\operatorname{cov} X, p)+\left(X^{2} / p^{4}\right)(\operatorname{var} p)$, $(\operatorname{cov} W, p)=(1 / p)(\operatorname{cov} X, p)-\left(X / p^{2}\right)(\operatorname{var} p)$, $(\operatorname{cov} W, Y)=(1 / p)(\operatorname{cov} X, Y)-\left(X / p^{2}\right)(\operatorname{cov} p, Y)$, $\mathrm{e}$

$\alpha x=\alpha^{* \prime} \alpha+\left(\mu_{p} / p\right)+(1 / p X)(\operatorname{cov} X, p)-\left(1 / p^{2}\right)(\operatorname{var} p)$.

Com

$J(W, Y, t) \equiv J(X / p, Y, t) \equiv V(X, Y, t)$,

Temos

$\left(J_{W W} / J_{W}\right)=\mathrm{p}\left(V_{X X} / V_{X}\right)$,

$\left(J_{W Y i} / J_{W}\right)=\left(V_{X Y i} / V_{X}\right)$ e

$\left(V_{X p} / V_{X}\right)=-(1 / p)-(x / p)\left(V_{X X} / V_{X}\right)$.

A equação (57) decorre, lançando as derivadas de $F$ ( $W, Y, t)$ nos termos das de $H(X, Y, t)$ e substituindo todas estas em (6). A taxa de juros nominal pode, então, ser identificada como o fluxo de payout nominal necessário para manter o valor nominal de um título idêntico a um, que é $\iota$ como dado em (58).

\section{Q.E.D.}

Uma comparação de (57) e (58) com (6) e (3) demonstra que a equação de taxa de juros e a equação fundamental de valoração têm exatamente a mesma forma quando todas as variáveis são expressas em termos nominais e quando todas as variáveis são expressas em termos reais. Usando os argumentos dados na prova da proposição, a taxa de juros nominal pode ser expressa em termos de riqueza real como

$$
\begin{aligned}
\text { (60) } & \iota=r+\left(\frac{1}{p}\right)\left[\mu_{p}-\left(\frac{-J_{W W}}{J_{W}}\right)(\operatorname{cov} W, p)-\sum_{i=1}^{k}\left(\frac{-J_{W Y}}{J_{W}}\right)\left(\operatorname{cov} Y_{i}, p\right)\right. \\
& \left.-\left(\frac{\operatorname{var} p}{p}\right)\right],
\end{aligned}
$$

onde $r$, a taxa de juros real, é como dado na equação (3). O termo $\left(\mu_{p} / p\right)$ é a taxa esperada de inflação. Osdemais termos podem, de modo geral, apresentar qualquer sinal, de modo que a taxa de juros nominal pode ser maior ou menor do que a soma da taxa de juros real e da taxa de inflação esperada. ${ }^{17}$

\section{CONSIDERAÇÕES FINAIS}

Neste estudo, aplicamos um modelo racional de precificação de ativos ao estudo da estrutura a termo das taxas de juros. Neste modelo, os preços atuais e as propriedades estocásticas de todos os direitos contingentes, inclusive debêntures, são derivados endogenamente. Antecipações, aversão ao risco, alternativas de investimento e preferências quanto ao momento do consumo representam papéis na determinação da estrutura a termo. O modelo inclui, assim, os principais fatores tradicionalmente mencionados de maneira condizente com o comportamento maximizador e com as expectativas racionais.

Por meio da exploração de exemplos específicos, para os preços das debêntures obtivemos soluções fechadas simples que dependem de variáveis econômicas observáveis e podem ser testadas. A combinação de princípios da precificação de ativos em equilíbrio intertemporal com uma modelagem adequada dos processos estocásticos subjacentes proporciona uma ferramenta poderosa para a derivação de teorias consistentes e potencialmente refutáveis. Este é o primeiro exercício de sua espécie e os métodos aqui desenvolvidos devem ter muitas aplicações além das aqui consideradas.

Em um artigo separado, Cox, Ingersoll e Ross (1981), usamos nossa abordagem para examinar alguns aspectos daquelas que podem ser consideradas as teorias tradicionais da estrutura a termo. Ali, demonstramos que algumas formas da hipótese clássica das expectativas condizem com nosso modelo de equilíbrio, seja o simples, seja em suas formas mais complexas, ao passo que as demais formas, de modo geral, não condizem. Demonstramos, também, a relação entre alguns modelos de equilíbrio em tempo contínuo e as teorias tradicionais que expressam as taxas spot esperadas futuras como combinações lineares das taxas spot passadas.

\section{AGRADECIMENTO}

Este artigo é uma versão ampliada da segunda metade de um working paper anterior de mesmo título. Agradecemos pelos úteis comentários e sugestões de muitos colegas, tanto de nossas próprias instituições quanto de outras. Essa pesquisa obteve o apoio da Dean Witter Foundation, do Center for Research in Security Prices e da National Science Foundation. 


\section{NOTAS}

(1) Agradecemos a Franco Modigliani por mencionar este ponto.

(2) Stiglitz (1970) enfatiza os aspectos de teoria da carteira (portfolio theory) associados a debêntures de diferentes vencimentos, como também o fazem Dieffenbach (1975), Long (1974) e Rubinstein (1976), que também incorporam as características de outros ativos. Modigliani e Shiller (1973) e Sargent (1972) destacaram a importância das antecipações racionais.

(3) Esse tipo de separabilidade foi demonstrado em diferentes contextos por Hakansson (1970), Merton (1971) e Samuelson (1969).

(4) Embora nossas premissas na presente seção não atendam a todas as restrições técnicas ao crescimento impostas à curva de utilidade e aos coeficientes da função de produção de Cox, Ingersoll e Ross (1985), quando combinadas, levam a um problema bem colocado com solução ótima que apresenta muitas propriedades úteis. A função de consumo ótima é $C^{*}(W, Y, t)=\left[\rho /\left(1-\exp \left(-\rho\left(t^{\prime}-t\right)\right)\right] W\right.$ e a curva de utilidade indireta tem a forma $J(W, Y, t)=a(t) \log W+b(t) Y+c(t)$, onde $a(t), b(t)$ e $c(t)$ são funções explicitamente determináveis do tempo.

(5) A condição $l^{\prime}\left(G G^{\prime}\right)^{-1} \alpha>1$, em conjunto com (13) e (14), garante que a taxa de juros seja sempre não negativa. Se $l^{\prime}\left(G G^{\prime}\right)^{-1} \alpha<1$, a taxa de juros será sempre não positiva.

(6) O equivalente em tempo discreto deste modelo foi testado por Wood (1964), embora, tendo ele se preocupado apenas com as expectativas, tenha deixado de especificar o termo de erro.

(7) Ver Feller (1951).

(8) Processos semelhantes a (17) foram estudados detidamente por Feller. A transformação laplaciana de (18) é dada em Feller (1951). Ver, em Johnson e Kotz (1970), uma descrição da distribuição quiquadrado não central. Oliver (1965) contém propriedades da função de Bessel modificada.

(9) Diversas cláusulas contratuais bastam para impedir o risco de inadimplência e fazer com que o valor de uma debênture independa da riqueza de seu vendedor. Por exemplo, os termos da debênture podem especificar que o vendedor deva recomprar a debênture ao preço dado por (23) sempre que sua riqueza caia para aquém de um determinado nível.

(10) Em nosso arcabouço, as circunstâncias mais importantes suficientes para que os preços das debêntures dependam exclusivamente da taxa de juros spot são: (i) os indivíduos têm aversão relativa constante ao risco, a incerteza da tecnologia pode ser descrita por uma só variável e a taxa de juros é uma função monotônica dessa variável, ou (ii) as mudanças da tecnologia são não estocásticas e a taxa de juros é função monotônica da riqueza

(11) Como o título objeto, uma debênture descontada, não realiza pagamentos durante a vida da opção, a análise de Merton (1973) implica que o exercício prematuro nunca seja ótimo e, portanto, calls americanas e européias têm o mesmo valor.

(12) Como vimos anteriormente, a origem somente é acessível se $\sigma 2>2 \kappa \theta$ Argumentos algo mais complexos podem ser usados para demonstrar que o modelo permanece inviável, mesmo que a origem seja inacessível.

(13) Entre os estudos que expressam as taxas spot futuras esperadas como combinações lineares das taxas spot atuais e passadas estão Bierwag e Grove (1967), Cagan (1956), De Leeuw (1965), Duesenberry (1958), Malkiel, (1966), Meiselman (1962), Modigliani e Shiller (1973), Modigliani e Sutch (1966), Van Horne (1965) e Wood (1964). Cox, Ingersoll e Ross (1981) examinam essa questão num ambiente de difusão.

(14) Ver esta representação em Brennan e Schwartz (1979).

(15) Se desejássemos fazer dos saldos reais de moeda um argumento na curva de utilidade indireta $U$, isso seria simples em nosso modelo. Uma política de oferta monetária maximizadora de utilidade dependeria apenas das variáveis de estado, da riqueza real e do tempo, de modo que o nível de preços induzido também dependeria unicamente dessas variáveis

(16) Slater (1965) dá as propriedades da função hipergeométrica confluente.

(17) Ver, em Fischer (1975), uma discussão correlata.

Artigo originalmente publicado por John C. Cox, Jonathan E. Ingersoll and Stephen A. Ross, sob o título "A Theory of the Term Structure of Interest Rates”, na Econometrica, v. 53, n. 2, p.385-408, 1985. Publicado com autorização da Econometric Society. (C) Econometric Society. http://www.econometricsociety.org

\section{REFERÊNCIAS}

BEJA, A. State preference and the riskless interest rate: a Markov model of capital markets. Review of Economic Studies, v. 46, n. 3, p. 435-446, 1979

BIERWAG, G. O.; GROVE, M. A. A model of the term structure of interest rates. Review of Economics and Statistics, v. 49, n. 1, p. 50-62, 1967.

BRENNAN, M. J.; SCHWARTZ, E. S. A continuous time approach to the pricing of bonds. Journal of Banking and Finance, v. 3, n. 2, p. 133-155, 1979. 


\section{JOHN C. COX - JONATHAN E. INGERSOLL JR. · STEPHEN A. ROSS}

CAGAN, P. The monetary dynamics of hyperinflation. In: FRIEDMAN, M. (Ed.). Studies in the Quantity Theory of Money. Chicago: University of Chicago Press, 1956.

COX, J. C.; INGERSOLL, J. E.; ROSS, S. A. A re-examination of traditional hypotheses about the term structure of interest rates. Journal of Finance, v. 36, n. 4, p. 769-799, 1981.

COX, J. C.; INGERSOLL, J. E.; ROSS, S. An intertemporal general equilibrium model of asset prices. Econometrica, v. 53, n. 2, p. 363-384, 1985.

CULBERTSON, J. M. The term structure of interest rates. Quarterly Journal of Economics, v, 71, n. 4, p. 485-517, Nov. 1957.

DE LEEUW, F. A model of financial behavior. In: DUESENBERRY, J. S., et al. The Brookings Quarterly Economic Model of the United States. Chicago: Rand McNally, 1965.

DIEFFENBACH, B. C. A quantitative theory of risk premiums on securities with an application to the term structure of interest rates. Econometica, v. 43, n. 3, p. 431-454, 1975.

DOTHAN, L. U. On the term structure of interest rates. Journal of Financial Economics, v. 6, n. 1, p. 59-69, Mar. 1978.

DUESENBERRY, J. A. Business Cycles and Economic Growth. New York: McGraw-Hill, 1958.

FELLER, W. Two singular diffusion problems. Annals of Mathematics, v. 54, n. 1, p. 173-182, 1951

FISCHER, S. The demand for index bonds. Journal of Political Economy, v. 83, n. 3 , p. $509-534,1975$.

GERMAN, M. B. A general theory of asset valuation under diffusion processes. University of California, Berkeley, Institute of Business and Economic Research, Working Paper, n. 50, 1977.

HAKANSSON, N. H. Optimal investment and consumption strategies under risk for a class of utility functions. Econometrica, v. 38, n. 5, p. 587-607, Sept. 1970.

HICKS, J. R. Value and Capital. 2nd ed. London: Oxford University Press, 1946.

JOHNSON, N. L.; KOTZ, S. Distributions in Statistics: Continuous Univariate Distributions - 2. Boston: Houghton Miffin, 1970.

LONG, J. B. Stock prices, inflation, and the term structure of interest rates. Journal of Financial Economics, v. 1, n. 2, p. 131-170, July 1974
MALKIEL, B. G. The Term Structure of Interest Rates: Expectations and Behavior Patterns. Princeton, NJ: Princeton University Press, 1966.

MEISELMAN, D. The Term Structure of Interest Rates. Englewood Cliffs, NJ: Prentice Hall, 1962.

MERTON. R. C. A dynamic general equilibrium model of the asset market and its application to the pricing of the capital structure of the firm. Massachusetts Institute of Technology, Sloan School of Management, Working Paper, n. 497-570, 1970

MERTON. R. C. Optimum consumption and portfolio rules in a continuous time model. Journal of Economic Theory, v. 3, n. 4, p. 373-413 1971.

MERTON. R. C. Theory of rational option pricing. Bell Journal of Economics and Management Science, v. 4, p. 141-183, 1973.

MODIGLIANI, F; SHILLER, R. J. Inflation, rational expectations and the term structure of interest rates. Econometrica, v. 40, sem número, p. 12 43,1973

MODIGLIANI, F; SUTCH, R. Innovations in interest rate policy. American Economic Review, v. 56, p. 178-197, May 1966

NELSON, C. R. The Term Structure of Interest Rates. New York: Basic Books, 1972

OLIVER, F. W. J. Bessel functions of integer order. In: ABRAMOWITZ, M. A.; STEGUN, A. I. Handbook of Mathematical Functions. New York: Dover, 1965.

RICHARD. S. F. An arbitrage model of the term structure of interest rates. Journal of Financial Economics, v. 6, n. 1, p. 33-57, 1978

ROLL, R. The Behavior of Interest Rates. New York: Basic Books, 1970.

ROLL, R. Investment diversification and bond maturity. Journal of Finance, v. 26, n. 1, p. $51-66,1971$

RUBINSTEIN, M. E. The valuation of uncertain income streams and the pricing of options. Bell Journal of Economics, v. 7, n. 2, p. 407-425, 1976.

SAMUELSON, P. A. Lifetime portfolio selection by dynamic stochastic programming. Review of Economics and Statistics, v. 51, n. 3, p. 239-246, 1959

SARGENT, T. J. Rational expectations and the term structure of interest rates. Journal of Money, Credit, and Banking, v. 4, n. 1, p. 74-97, 1972. 
SLATER, L. J. Confluent hypergeometric functions. In: ABRAMOWITZ, M.; STEGUN, I. A. (Eds.). Handbook of Mathematical Functions. New York: Dover, 1965.

STIGLITZ, J. E. A consumption-oriented theory of demand for financial assets and the term structure of interest rates. Review of Economic Studies, v. 37, n. 3, p. 321-351, 1970.
VAN HORNE, J. C. Interest-rate risk and the term structure of interest rates. Journal of Political Economy, v. 73, n. 6, p. 344-351, 1965.

VASICEK, O. A. An equilibrium characterization of the term structure. Journal of Financial Economics, v. 5, n. 2, p. 177-188, Nov. 1977.

WOOD, J. H. The expectations hypothesis, the yield curve and monetary policy. Quarterly Journal of Economics, v. 78, n. 1, p. 457-470, 1964.

Artigo convidado. Aprovado em 16.03.2007.

\section{John C. Cox}

Professor de Finanças Econômicas na MIT Sloan School of Management.

Interesses de pesquisa nas áreas de finanças corporativas; teoria financeira; gestão de carteira.

E-mail: rbourke@mit.edu.

Endereço: Memorial Drive, 50, 02142, Cambridge, Massachusetts, USA.

\section{Jonathan E. Ingersoll Jr.}

Professor de Negócios Internacionais e Finanças da Yale School of Management.

Interesses de pesquisa nas áreas de avaliação de ativos, precificação de ativos e estrutura a termo de taxas de juros.

E-mail: jonathan.ingersoll@yale.edu.

Endereço: Caixa Postal 208200, 06520-8200, New Haven, CT, USA.

\section{Stephen A. Ross}

Professor de Finanças Econômicas na MIT Sloan School of Management.

Interesses de pesquisa nas áreas de finanças corporativas, mercados financeiros e arbitrage pricing theory (APT).

E-mail: sgrosv@mit.edu

Endereço: Memorial Drive, 50, 02142, Cambridge, Massachusetts, USA. 